\title{
Sex-specific features of emphysema among current and former smokers with COPD
}

\author{
Megan Hardin ${ }^{1,2}$, Marilyn Foreman ${ }^{3}$, Mark T. Dransfield ${ }^{4}$, Nadia Hansel ${ }^{5}$, \\ MeiLan K. Han ${ }^{6}$, Michael H. Cho ${ }^{1,2}$, Surya P. Bhatt ${ }^{7}$, Joe Ramsdell ${ }^{8}$, \\ David Lynch ${ }^{9}$, Jeffrey L. Curtis ${ }^{6,10}$, Edwin K. Silverman ${ }^{1,2}$, George Washko ${ }^{2}$ and \\ Dawn DeMeo ${ }^{1,2}$ for the COPDGene Investigators
}

\begin{abstract}
Affiliations: ${ }^{1}$ Channing Division of Network Medicine, Brigham and Women's Hospital, Harvard Medical School, Boston, MA, USA. 'Division of Pulmonary and Critical Care Medicine, Brigham and Women's Hospital, Harvard Medical School, Boston, MA, USA. ${ }^{3}$ Morehouse School of Medicine, Department of Internal Medicine, Atlanta, GA, USA. ${ }^{4}$ Division of Pulmonary, Allergy, and Critical Care Medicine, University of Alabama at Birmingham, Birmingham, AL, USA. ${ }^{5}$ Johns Hopkins School of Medicine, Baltimore, MD, USA. ${ }^{6}$ University of Michigan Health System, Ann Arbor, MI, USA. ${ }^{7}$ University of Alabama, Birmingham, AL, USA. ${ }^{8}$ Division of Internal Medicine, University of California, San Diego, CA, USA. 'Dept of Radiology, National Jewish Health, Denver, CO, USA. ${ }^{10}$ VA Ann Arbor Healthcare System, Ann Arbor, MI, USA.
\end{abstract}

Correspondence: Megan Hardin, Channing Division of Network Medicine, 181 Longwood Avenue, Boston, MA 02116, USA. E-mail: remehdachanning.harvard.edu

ABSTRACT Recent studies suggest that males with chronic obstructive pulmonary disease (COPD) have more emphysema than females. It is not known if these differences persist across degrees of COPD severity. Our aim was to identify sex-specific differences in quantitative emphysema within COPD subgroups based on COPD severity.

We included non-Hispanic white and African-American subjects from the COPDGene study with at least 10 pack-years of smoking and COPD Global Initiative for Chronic Obstructive Lung Disease (GOLD) spirometry grade II or greater. We examined sex-specific differences in log-transformed emphysema (log per cent low-attenuation area (\%LAA)) by GOLD spirometry grade among subjects with early-onset COPD ( $<55$ years old) and advanced emphysema ( $>25 \%$ emphysema).

Compared with females, males had higher log \%LAA: overall (1.97 \pm 1.4 versus $1.69 \pm 1.6, \beta=0.32(0.04)$, $\left.\mathrm{p}=1.34 \times 10^{-14}\right)$, and among non-Hispanic white $\left(\mathrm{p}=8.37 \times 10^{-14}\right)$ and African-American subjects $(\mathrm{p}=0.002)$. Females with early-onset COPD, severe emphysema and GOLD grade IV COPD had similar emphysema as males, but markedly fewer pack-years smoking (early-onset, $\mathrm{p}=0.01$; severe emphysema and GOLD grade IV, $\mathrm{p}<0.001$ ).

This study identifies subsets of female smokers with COPD who are particularly susceptible to parenchymal destruction.

@ERSpublications

In severe COPD subgroups, females have greater susceptibility to smoking-related parenchymal lung damage/emphysema http://ow.ly/Stqyz

For editorial comments see Eur Respir J 2016; 47: 19-22 [DOI: 10.1183/13993003.01781-2015].

This article has supplementary material available from erj.ersjournals.com

Received: June 232015 | Accepted after revision: Sept 05 2015 | First published online: Nov 52015

Support statement: R01 HL089438, K12 HL120004, R01 HL089856, R01 HL089897, P01 HL105339, 2013 Sheila J. Goodnight, MD, FCCP Clinical Research Grant in Women's Lung Health. The COPDGene project described was supported by Award Number R01 HL089897 and Award Number R01 HL089856 from the National Heart, Lung, and Blood Institute. The content is solely the responsibility of the authors and does not necessarily represent the official views of the National Heart, Lung, and Blood Institute or the National Institutes of Health. The COPDGene project is also supported by the COPD Foundation through contributions made to an Industry Advisory Board comprised of AstraZeneca, Boehringer Ingelheim, Novartis, Pfizer, Siemens, Sunovion and GlaxoSmithKline. Funding information for this article has been deposited with FundRef.

Conflict of interest: Disclosures can be found alongside the online version of this article at erj.ersjournals.com

The content of this work is copyright of the authors or their employers. Design and branding are copyright @ERS 2016. 


\section{Introduction}

Chronic obstructive pulmonary disease (COPD) is the third leading cause of death in the USA, with $>15$ million Americans affected [1]. COPD prevalence and mortality among females have risen rapidly in the last several decades, and are now equal to COPD prevalence and mortality among males [2, 3]. Multiple studies suggest that females experience greater lung function decline than males for the same amount of cigarette exposure [4-7]. Although the prevalence of COPD among African-Americans is less than among non-Hispanic whites [2], African-Americans demonstrate greater lung function decline in response to cigarette smoke, with some studies suggesting that African-American females are particularly susceptible to tobacco smoke [4]. Despite the growing recognition that COPD is not just a male disease, research into sex-specific features of COPD has been limited and is needed [8].

Radiographic emphysema is a clinically relevant COPD phenotype that correlates with loss of lung function $[9,10]$, expiratory airflow limitation [11], decreased diffusing capacity [12], decreased exercise capacity [13, 14], increased respiratory exacerbations [15] and respiratory mortality [16-18]. Identifying sex differences in emphysema may lead to insights into sex-specific differences in COPD susceptibility, severity, comorbidities and treatments.

Several prior studies have compared cross-sectional radiographic emphysema between males and females. These studies have suggested that, as a group, males with COPD have more overall emphysema than females for the same degree of airflow obstruction [19-22]. Based on these studies, it would appear that females are less sensitive to parenchymal damage from cigarette smoke than males. This would run counter to evidence suggesting that females have greater lung function decline in response to smoking [5]. However, COPD is a complex disease with multiple subphenotypes. These studies were underpowered to identify whether the trend for greater emphysema among male COPD subjects persists among severe COPD subtypes, including severe emphysema and advanced or early-onset COPD. In addition, these trends have not been fully explored among African-American populations. One study among a subset of subjects from COPDGene demonstrated that African-Americans had less overall emphysema than non-Hispanic whites [23]. A further analysis of emphysema differences within these subgroups and across different races is an essential step towards understanding how COPD can develop and progress by sex, insights which may inform sex-specific clinical care of patients.

This study responds to a call from the National Institutes of Health for more investigation into the sex-based differences in disease [24] and, in particular, the need for increased investigation into the sex-specific features of COPD. The goals of this study were to investigate sex-specific differences in emphysema across a large population of COPD subjects with a comprehensive range of lung function, and to investigate sex-specific associations between cigarette smoke dosage and emphysema. We hypothesised that male predominance of emphysema would not persist among severe COPD subgroups.

\section{Materials and methods \\ Subjects}

All subjects signed informed consent, and Institutional Review Board approval was obtained at Brigham and Women's Hospital (2007P000554; Partners Human Research Committee) and all study sites (online supplementary table S11). All subjects were non-Hispanic white or African-American current or former smoking participants in the COPDGene study [25]. All subjects completed a modified American Thoracic Society (ATS) respiratory questionnaire and standardised pulmonary function testing. Our main analysis was among subjects with COPD by ATS/European Respiratory Society criteria (post-bronchodilator forced expiratory volume in $1 \mathrm{~s}$ (FEV1)/forced vital capacity (FVC) $<70 \%, \mathrm{FEV}_{1}<80 \%$ predicted) and Global Initiative for Chronic Obstructive Lung Disease (GOLD) spirometry grade II-IV, based on the GOLD 2006 classifications scheme. We examined per cent low-attenuation area (\%LAA) differences among all subjects and stratified by race, GOLD spirometry grade, among those subjects with early-onset COPD, and severe emphysema. Early-onset COPD subjects were $<55$ years old with $\mathrm{FEV}_{1}<50 \%$ predicted [26]. Severe emphysema was defined as $>25 \%$ low-attenuation area (LAA) on computed tomography (CT) [27]. We additionally investigated smoking controls from COPDGene (current or former smokers with $>10$ pack-years smoking history and normal spirometry).

\section{Radiographic measurements}

Quantitative emphysema (\%LAA) was measured using inspiratory CT scans performed at each clinical centre and analysed with Slicer software $[15,25]$. The total emphysema (\%LAA) was defined as the percentage of lung with LAAs less than -950 Hounsfield units (HU) [28]. We additionally examined \%LAA at $-910 \mathrm{HU}$. 


\section{Statistical analysis}

All analyses were performed using R (version 2.15.1; https://www.r-project.org/). We compared means of continuous variables using t-tests and binary variables using Pearson Chi-squared analysis. We used Pearson correlations to measure the correlation between pack-years of smoking and emphysema by sex. We transformed \%LAA to the natural log scale [29] and performed univariate comparisons of log \%LAA using t-tests. We performed logistic regression for log \%LAA, adjusting for age, pack-years smoking, current smoking status and body mass index (BMI). Analyses including both non-Hispanic white and African-American subjects were additionally adjusted for race. We adjusted for CT scanner model using indicator variables for each CT scanner model included in COPDGene [30]. Logistic regressions for mean \%LAA are additionally presented in the online supplementary material. We performed a sensitivity analysis among subjects scanned with a single scanner (Siemens Definition), comparing log \%LAA between males and females by sex and race (see online supplementary material). We performed an additional analysis to examine sex differences in additional exposures and their impact on emphysema, including age at starting smoking, adult second-hand smoke exposure, and occupational smoke, dust and fumes exposure. We performed backwards selection to identify variables associated with log \%LAA and performed multivariate linear regression for the outcome log \% LAA additionally adjusting for these covariates.

\section{Results}

\section{Subject demographics}

A total of 3690 subjects with GOLD spirometry grade II-IV COPD were included in the analyses (table 1). Of these subjects, 836 were African-Americans. Both non-Hispanic white and African-American female subjects had fewer pack-years of smoking than males (non-Hispanic whites: $50.3 \pm 23.7$ versus $60.7 \pm 30.2$ pack-years, $\mathrm{p}<0.001$; African-Americans: $38.7 \pm 21.3$ versus $45.6 \pm 24.0$ pack-years, $\mathrm{p}<0.001$ ).

\section{Quantitative emphysema}

In multivariate analyses comparing log \%LAA between males and females with COPD, stratified by race, and adjusted for age, BMI, pack-years, current smoking status and CT scanner type (figure 1 and table 2), males had greater emphysema than females, both among non-Hispanic whites $(2.06 \pm 1.3$ versus $1.79 \pm 1.5$, $\left.\beta=0.34 \pm 0.05, p=8.37 \times 10^{-14}\right)$ and African-Americans ( $1.63 \pm 1.5$ versus $1.31 \pm 1.7, \beta=0.31 \pm 0.10, p=0.002$ ). This trend persisted with the mean \%LAA outcome (online supplementary table S1) and among subjects scanned with a single CT scanner type (online supplementary table S2). A similar trend was observed when comparing the outcome of \%LAA at $-910 \mathrm{HU}$ (online supplementary table S3). The sex-specific difference in log \%LAA was also observed among 4388 current or former smokers with normal lung function (online supplementary tables S4 and S5).

\section{TABLE 1 Demographic data}

\begin{tabular}{|c|c|c|c|c|c|c|}
\hline & \multicolumn{3}{|c|}{ Non-Hispanic white } & \multicolumn{3}{|c|}{ African-American } \\
\hline & Male & Female & p-value ${ }^{\#}$ & Male & Female & p-value ${ }^{\#}$ \\
\hline COPD subjects $n$ & 1593 & 1261 & & 459 & 377 & \\
\hline Age years & $65.1 \pm 8.2$ & $64.1 \pm 8.3$ & 0.002 & $58.8 \pm 8.0$ & $59.2 \pm 8.3$ & 0.46 \\
\hline Pack-years & $60.7 \pm 30.2$ & $50.3 \pm 23.7$ & $<0.001$ & $45.6 \pm 24.0$ & $38.7 \pm 21.3$ & $<0.001$ \\
\hline Current smoker & 556 (34.9) & $436(34.6)$ & 0.89 & $301(65.6)$ & 208 (55.2) & 0.003 \\
\hline BMI $\mathrm{kg} \cdot \mathrm{m}^{-2}$ & $28.4 \pm 5.6$ & $27.7 \pm 6.7$ & 0.008 & $26.7 \pm 6.0$ & $29.5 \pm 7.3$ & $<0.001$ \\
\hline FEV $1 \%$ predicted & $49.12 \pm 18.1$ & $50.28 \pm 17.8$ & 0.09 & $51.79 \pm 18.2$ & $52.81 \pm 17.2$ & 0.41 \\
\hline FEV $1 / F V C$ & $0.48 \pm 0.1$ & $0.50 \pm 0.1$ & $<0.001$ & $0.52 \pm 0.1$ & $0.53 \pm 0.1$ & 0.13 \\
\hline GOLD II & 776 (48.7) & $668(53.0)$ & & $252(54.9)$ & $226(60.0)$ & \\
\hline GOLD II & 531 (33.3) & $388(30.8)$ & & $136(29.6)$ & $107(28.4)$ & \\
\hline GOLD IV & 286 (18.0) & 205 (16.3) & & 71 (15.5) & $44(11.7)$ & \\
\hline$\%$ LAA & $14.2 \pm 12.9$ & $12.9 \pm 12.8$ & 0.008 & $11.4 \pm 12.5$ & $9.7 \pm 11.7$ & 0.06 \\
\hline Log \%LAA & $2.06 \pm 1.3$ & $1.79 \pm 1.5$ & $<0.001$ & $1.63 \pm 1.5$ & $1.31 \pm 1.7$ & 0.006 \\
\hline Early-onset COPD & $78(4.9)$ & $81(6.4)$ & 0.09 & $52(11.3)$ & $49(13.0)$ & 0.53 \\
\hline Emphysema predominant ${ }^{+}$ & $304(19.1)$ & $216(17.1)$ & 0.20 & $68(14.8)$ & 39 (10.3) & 0.07 \\
\hline
\end{tabular}

Data are presented as mean \pm SD or $\mathrm{n}(\%)$, unless otherwise stated. COPD: chronic obstructive pulmonary disease; BMI: body mass index; FEV1: forced expiratory volume in $1 \mathrm{~s}$; FVC: forced vital capacity; GOLD: Global Initiative for Chronic Obstructive Lung Disease; \%LAA: per cent low attenuation area. \#: univariate comparison of non-Hispanic white and African-American males versus females, respectively; ${ }^{n}:<55$ years old, GOLD grade III or IV; ${ }^{+}$: subjects with $\geqslant 25 \%$ emphysema on CT. 


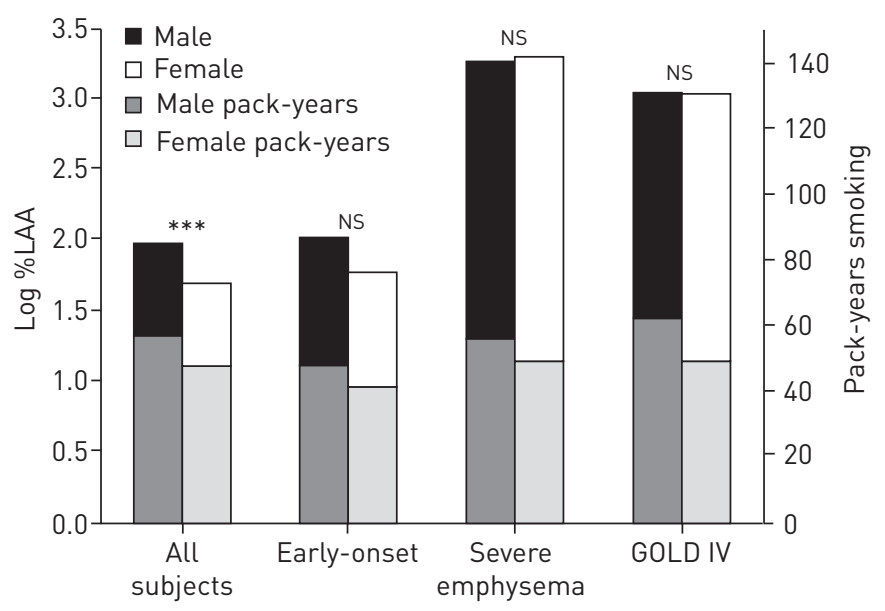

FIGURE 1 Log per cent low-attenuation area (\%LAA) and pack-years across severe chronic obstructive pulmonary disease (COPD) phenotypes. All subjects: non-Hispanic white and African-American subjects with COPD; early-onset: early-onset COPD subjects; severe emphysema: COPD subjects with >25\% LAA; GOLD IV: all non-Hispanic white and African-American subjects with Global Initiative for Chronic Obstructive Lung Disease grade IV. p-values from the generalised linear model adjusted for age, race, body mass index, pack-years smoking, current smoking status and computed tomography scanner type. ${ }^{* * *}$ : $p<0.001$; NS: nonsignificant $(p>0.05) . p<0.01$ for the difference in pack-years by sex for each COPD subtype.

\section{Pack-years of smoking and emphysema}

Overall, males had greater pack-years of smoking than females (table 1). Pack-years of smoking was significantly but weakly correlated with log \%LAA in males and females (males: $r=0.12, p<0.001$; females: $\mathrm{r}=0.08, \mathrm{p}=0.002$ ). Among all COPD subjects, there was not a significant interaction between sex and pack-years for the outcome log \%LAA ( $\mathrm{p}=0.69$ for non-Hispanic whites, $\mathrm{p}=0.97$ for African-Americans and $\mathrm{p}=0.78$ for all subjects). However, the interaction between sex and current smoking status was significant for this outcome among all subjects $(p=0.004)$ and non-Hispanic whites $(p=0.01)$, but only a trend among African-Americans $(p=0.06)$. Among the smoking controls, males demonstrated more emphysema per pack-year smoked than females $(\mathrm{p}<0.001)$, but there was no difference in the amount of emphysema per pack-year smoked among all of the COPD subjects ( $\mathrm{p}=0.74)$ (online supplementary table S6).

\section{Quantitative CT traits and the sex-specific relationship with lung function}

To evaluate whether the relationship between amount of emphysema and lung function differed by sex, we examined sex-specific differences in log \%LAA by GOLD spirometry grade (table 3 and figure 2). Log \%LAA increased with each GOLD spirometry grade in males and females. The disparity between the sexes in log \%LAA was seen most strongly among the GOLD II subjects (males: $1.39 \pm 1.3 \%$ versus females: $1.09 \pm 1.5 \%$, $\beta=0.36 \pm 0.05, p<0.001$ ) and decreased to nonsignificant among subjects with GOLD grade III and IV COPD. Similarly, for grade IV COPD, there was no difference in log \%LAA between males and females among all subjects combined ( $3.05 \pm 0.85$ versus $3.03 \pm 0.91, \beta=0.12 \pm 0.06, \mathrm{p}=0.07)$ or among African-American subjects. Among non-Hispanic white subjects, males with GOLD grade IV COPD demonstrated statistically significant but clinically minimal difference in $\log \%$ LAA (males: $3.08 \pm 0.8$ versus females: $3.02 \pm 1.0, \beta=0.17 \pm 0.07$, $\mathrm{p}=0.02$ ). Males had significantly more pack-years smoking than females overall (table 1) and at all GOLD

\begin{tabular}{lcccc} 
TABLE 2 Log per cent low-attenuation area (\%LAA) by sex and race & \\
& Male & Female & $\boldsymbol{\beta} \pm$ SE & p-value \\
\hline All subjects & $1.97 \pm 1.4$ & $1.69 \pm 1.6$ & $0.32 \pm 0.04$ & $<0.001^{\#}$ \\
Non-Hispanic whites & $2.06 \pm 1.3$ & $1.79 \pm 1.5$ & $0.34 \pm 0.05$ & $<0.001^{\text {甲 }}$ \\
African-Americans & $1.63 \pm 1.5$ & $1.31 \pm 1.7$ & $0.31 \pm 0.1$ & $0.002^{+}$
\end{tabular}

Data are presented as mean $\pm \mathrm{SD}$, unless otherwise stated. Including all subjects with Global Initiative for Chronic Obstructive Lung Disease grade II-IV chronic obstructive pulmonary disease. Adjusted for age, pack-years, current smoking status, body mass index and computed tomography scanner. Log \%LAA: emphysema (\%LAA) log-transformed for normality; $\beta$ : difference for male subjects compared with female subjects. Interaction between current smoking and sex on log \%LAA: ": all subjects, $p=0.004 ;{ }^{n}$ : non-Hispanic white subjects, $p=0.01$; ${ }^{+}$: African-American subjects, $\mathrm{p}=0.06$. 


\begin{tabular}{|c|c|c|c|c|}
\hline & Male & Female & $\beta \pm S E$ & p-value \\
\hline \multicolumn{5}{|c|}{ All subjects } \\
\hline II & $1.39 \pm 1.28$ & $1.09 \pm 1.47$ & $0.36 \pm 0.05$ & $<0.001$ \\
\hline III & $2.32 \pm 1.25$ & $2.14 \pm 1.39$ & $0.25 \pm 0.07$ & 0.001 \\
\hline IV & $3.05 \pm 0.85$ & $3.03 \pm 0.91$ & $0.12 \pm 0.06$ & 0.07 \\
\hline \multicolumn{5}{|c|}{ Non-Hispanic whites } \\
\hline II & $1.49 \pm 1.24$ & $1.20 \pm 1.43$ & $0.37 \pm 0.06$ & $<0.001$ \\
\hline III & $2.37 \pm 1.21$ & $2.22 \pm 1.37$ & $0.30 \pm 0.07$ & $<0.001$ \\
\hline IV & $3.08 \pm 0.82$ & $3.02 \pm 0.95$ & $0.17 \pm 0.07$ & 0.02 \\
\hline \multicolumn{5}{|c|}{ African-Americans } \\
\hline II & $2.34 \pm 1.07$ & $2.1 \pm 1.27$ & $0.35 \pm 0.10$ & $<0.001$ \\
\hline III & $2.13 \pm 1.4$ & $1.87 \pm 1.43$ & $0.24 \pm 0.17$ & 0.14 \\
\hline IV & $2.39 \pm 1.13$ & $2.1 \pm 1.28$ & $0.27 \pm 0.19$ & 0.14 \\
\hline
\end{tabular}

Data are presented as mean \pm SD, unless otherwise stated. Log \%LAA: emphysema (\%LAA) log-transformed for normality; $\beta$ : difference for male subjects compared with female subjects. Adjusted for age, sex, pack-years, body mass index, current smoking status and computed tomography scanner. "All subjects" additionally adjusted for race.

stages, including GOLD IV (males: $61.8 \pm 31.4$ versus females: $49.2 \pm 24.3, \beta=11.1 \pm 2.3, p<0.001$ ). These same trends persisted when comparing mean \%LAA by sex among each GOLD group (online supplementary table S7). When baseline FEV1 was included in the model examining the relationship between log \%LAA and sex, males continued to have significantly more emphysema than females $(\beta=0.7 \pm 0.04, p<0.001)$.

\section{Sex-specific emphysema patterns among early-onset COPD subjects}

There were 260 subjects with early-onset COPD (table 4). Females with early-onset COPD had statistically similar $\log \%$ LAA as males (females: $1.78 \pm 1.6$ versus males: $2.06 \pm 1.4, \beta=0.13 \pm 0.18, p=0.47$ ). As seen previously [26], females demonstrated similar lung function as measured by FEV1 percent predicted (females: $35.52 \pm 10.6 \%$ versus males: $35.52 \pm 9.8 \%, \mathrm{p}=0.99$ ), but fewer pack-years of smoking (females: $41.0 \pm 20.0$ versus males: $48.2 \pm 25.4$ pack-years, $\mathrm{p}=0.01)$. There was no interaction between sex and pack-years smoking $(\mathrm{p}=0.42)$. These same trends persisted when comparing mean \%LAA (online supplementary table S8).

\section{Sex-specific emphysema patterns among emphysema-predominant subjects}

There were 627 subjects with $>25 \%$ LAA on chest CT scans (table 4). There were more males than females in this group. Males and females with emphysema predominance were similar in age and lung function (table 4). However, the females in this group had significantly fewer pack-years than males (females: $49.1 \pm 22.1$ versus males: $56.3 \pm 25.8$ pack-years, $\mathrm{p}<0.001$ ), despite similar log \%LAA (females: $3.54 \pm 0.2$ versus males: $3.54 \pm 0.2$, $\mathrm{p}=0.19$ ). This same trend was seen when comparing mean \%LAA (online supplementary table S8).

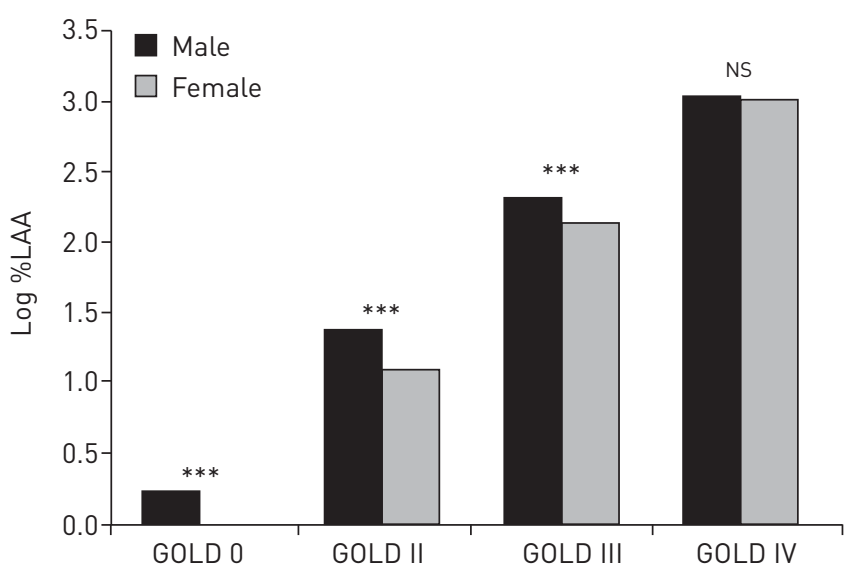

FIGURE 2 Log per cent lowattenuation area (\%LAA) by spirometric Global Initiative for Chronic Obstructive Lung Disease (GOLD) grade. p-values from generalised linear models adjusted for age, race, body mass index, pack-years smoking, current smoking status and computed tomography scanner type. $* * *$ : $p<0.001$; NS: nonsignificant ( $p>0.05$ ). 
TABLE 4 Log per cent low-attenuation area (\%LAA) among severe chronic obstructive pulmonary disease (COPD) subtypes

\begin{tabular}{|c|c|c|c|c|c|c|c|c|}
\hline & \multicolumn{4}{|c|}{ Early-onset COPD } & \multicolumn{4}{|c|}{ Severe emphysema } \\
\hline & Male & Female & $\beta \pm S E$ & p-value & Male & Female & $\beta \pm \mathrm{SE}$ & p-value \\
\hline Subjects $\mathrm{n}$ & 130 & 130 & & & 372 & 255 & & \\
\hline Pack-years & $48.2 \pm 25.4$ & $41.0 \pm 20.0$ & & 0.01 & $56.31 \pm 25.8$ & $49.11 \pm 22.1$ & & $<0.001$ \\
\hline FEV $1 \%$ predicted & $35.52 \pm 9.8$ & $35.52 \pm 10.6$ & & 0.99 & $33.5 \pm 14.1$ & $32.93 \pm 13.5$ & & 0.61 \\
\hline Log \%LAA & $2.06 \pm 1.4$ & $1.78 \pm 1.6$ & $0.13 \pm 0.18$ & 0.47 & $3.54 \pm 0.2$ & $3.54 \pm 0.2$ & $0.022 \pm 0.02$ & 0.19 \\
\hline
\end{tabular}

Data are presented as mean \pm SD, unless otherwise stated. Log \%LAA: emphysema (\%LAA) log-transformed for normality; early-onset COPD: COPD subjects with Global Initiative for Chronic Obstructive Lung Disease grade III and IV and age <55 years; severe emphysema: $>25 \%$ LAA; FEV1: forced expiratory volume in $1 \mathrm{~s}$. Univariate comparisons performed for age, pack-years and FEV1 \% predicted. \%LAA adjusted for age, race, pack-years, smoking status, body mass index and computed tomography scanner.

\section{Environmental exposures}

We examined the relationship between environmental exposures and log \%LAA. Overall, males had greater second-hand smoke exposure, including smoke exposure at work, were more likely to have worked in a job with dust or fumes and were more likely to be currently working in a job with dust or fumes (online supplementary table S9). In a multivariate analysis, passive smoke $(\beta=0.003 \pm 0.001, p=0.02)$ and fumes exposure at work $(\beta=0.20 \pm 0.05, p<0.001)$ were significantly associated with $\log \% \mathrm{LAA}$, and years of second-hand smoke exposure at work approached significance $(\beta=0.003 \pm 0.001, p<0.07)$. The addition of these covariates to the multivariate regression examining log \%LAA did not change the general trends noted in our main analysis. (online supplementary table S10). We additionally included maternal smoking and maternal history of COPD in our model examining the relationship between sex and \%LAA among all subjects and early-onset subjects; the inclusion of these variables did not impact the overall trends of our results.

\section{Discussion}

In this analysis of COPDGene subjects, we demonstrate that although males displayed greater radiographic emphysema extent than females overall, females in three phenotypic subgroups (early-onset COPD, severe emphysema and GOLD grade IV COPD) had comparable radiographic emphysema, despite fewer pack-years of smoking. These findings suggest a subset of female smokers who are particularly susceptible to parenchymal lung destruction. Our results significantly extend previous studies showing that among COPD subjects in aggregate, males tend to have an emphysema-predominant phenotype and females have airway involvement $[19,21,22,31]$. Those prior studies were limited by sample size or subject ascertainment and therefore had limited generalisability to specific subsets of COPD subjects. Given the increasing recognition that radiographic emphysema is a clinically meaningful COPD phenotype, even in the absence of airflow obstruction, our findings fill an important knowledge gap on sex-based differences in lung disease.

Early-onset COPD is an extreme COPD subgroup, and may prove especially useful for investigating the role of sex-specific genetic factors in COPD development and progression. Prior studies have suggested a distinct pathogenesis for early-onset COPD encompassing sex-specific immune responses, genetic or epigenetic effects [26]. Subjects with early-onset COPD have been shown to be predominantly female [32], a finding replicated in an analysis of the first 2500 subjects from COPDGene [26]. Linkage and familial aggregation studies have identified potential genetic risk factors for early-onset COPD [33-35]; the finding of markedly reduced lung function in female first-degree relatives of probands with early-onset COPD [36] suggests that females may be particularly susceptible to this COPD subtype. Our finding that females with early-onset COPD have equivalent emphysema with fewer pack-years of smoking is consistent with these suggestions. In contrast to our findings, however, САMP et al. [19] examined probands and their siblings with COPD from the International COPD Genetics Network study. In that population, they found that more males had an emphysema pattern and females an airway predominant pattern. Although probands in that study were selected for COPD occurring before age 65, the subjects overall differed from those included here, including fewer subjects with severe COPD.

Our findings among the emphysema-predominant subgroup differ from the observations among subjects with severe emphysema being considered for lung volume reduction surgery from the National Emphysema Treatment Trial (NETT). MarTinEz et al. [21] demonstrated that among these subjects, males had more emphysema than females, as measured by whole-lung per cent emphysema on CT scan. However, their study contrasts with COPDGene in several ways. NETT study subjects were defined by the presence of severe COPD (FEV $1<45 \%$ predicted) and subjects considered high-risk for lung-volume reduction surgery were excluded, including those with very severe COPD (FEV $1<20 \%$ predicted). NETT subjects had 
significant pack-years smoking history with a mean of 71 pack-years in males and 55 pack-years in females. Among COPDGene subjects, we observed that the sex-specific differences in emphysema were less distinct among the heaviest smokers, as well as those with the most severe reductions in lung function.

Our finding that the sex-based differences were most striking at early grades of the disease (GOLD II), but equivalent by later-grade disease, is important because it may suggest that sex-based emphysema patterns begin early in the disease process but later progress differently in males and females depending on additional susceptibility factors. This result contrasts with a prior study of 396 subjects with COPD GOLD II-IV from the National Lung Screening Trial [31]. In that study, males overall had greater emphysema than females. However, the differences in per cent emphysema were not significantly different between males and females with GOLD II COPD, but were for GOLD III/IV males and females. COPDGene includes more subjects with severe COPD and is better powered to examine differences in emphysema among this subset of subjects. In addition, this current study accounts for pack-years and smoking status when comparing emphysema between the sexes.

Our study also demonstrated similar, but less marked, sex-based differences in COPD among African-American subjects, relative to non-Hispanic white sex-based differences. In a previous analysis among the first 2500 subjects from the COPDGene study, HANSEL et al. [23] observed that African-American subjects had less overall emphysema, as well as less lower-lobe emphysema, than non-Hispanic whites. We demonstrate that the sex-based differences in emphysema are less marked in African-American subjects, but the trend is still present. It is of note that in our study, African-American females had higher BMI than African-American males, while the converse was seen in non-Hispanic whites. This trend mirrors that seen among US adults as a whole [37]. Theoretically, increased BMI could lead to decreased effective radiation dose and artefactually increase \%LAA measurement [30,38]; however, we have adjusted for BMI in our model.

We chose to use log \%LAA as our measure of emphysema. This end-point is determined by calculating the percentage of voxels on the chest CT that are below a certain limit, in this case less than $-950 \mathrm{HU}$. The Slicer method is a computer algorithm and therefore not subject to interobserver variability. However, while it provides a quantitative assessment of overall emphysema, it does not yet parse subtypes such as panlobular, paraseptal and centrilobular emphysema. Several other methods for calculating emphysema have been developed $[39,40]$ and this is an area of active research. While analysing sex-based differences in all of these outcomes is of interest, and is an area for future work, for this current analysis we chose an established and validated method.

Our study has several limitations. First, because \%LAA is a highly skewed variable, we log-transformed this outcome as has been done previously [29]; however, we recognise that log-transformed values are less straightforward. We have included the analysis of untransformed mean emphysema for our main analyses (see online supplementary material). Second, we attempted to identify sex-specific differences in emphysema related to smoking history. Our findings were mixed, which might reflect either that quantitative emphysema is poorly correlated with pack-years of smoking history or that pack-years is not the best metric to characterise cigarette smoke exposure. The pack-years variable is a best estimation of prior cigarette smoke exposure; however, this variable is limited in the ability to assess the impact of smoke exposure as it assumes a continuous exposure and linear dosage relationship. Additionally, one pack of cigarettes could represent a different dose for males and females as females have smaller lung size. Differences in emphysema between males and females may be related to sex-specific differences in the response to cigarette smoke: \%LAA is correlated with decreased lung function [9] and females with COPD demonstrated greater lung function decline per cigarette smoked than males [4,5]. Although cigarette smoke is clearly the most important factor in COPD development in industrialised nations, other exposures may impact the progression of emphysema. We attempted to examine the role of such additional exposures, including second-hand smoke, and occupational dust and fumes. Although females exposed to biomass fuels have demonstrated distinct emphysema patterns from females with lung function decline from smoking [41], our cohort was ascertained with an emphasis on current and former smokers. These results may be less generalisable to areas where biomass fuel exposure is more prevalent. While males in our study had greater exposures to second-hand smoke, and occupational dust and fumes, the general trends from our main analysis remained significant after adjusting for additional exposures. Third, we cannot exclude the possibility of selection bias, i.e. that females with lung disease and fewer pack-years of smoking history may have been more willing to enrol in the COPDGene study. Finally, body composition can impact radiographic assessment of emphysema and sex-based differences in subcutaneous tissue could impact our findings; to overcome this, as has been done in prior studies, we adjusted for BMI as a covariate in our regression models. The potential for differential attenuation by breast tissue remains. Current CT scan techniques do not account for breast tissue attenuation and this is an area for methods development.

The apparent sexual dimorphism in radiographic emphysema may relate to sex-specific differences in the biologic impact of cigarette smoke dosage. The finding that females demonstrate equivalent emphysema 
despite fewer pack-years of smoking in the early-onset and emphysema-predominant subsets suggests an increased susceptibility among females in these groups to parenchymal injury associated with smoking. COPD is a complex disorder impacted by heterogeneous susceptibility factors. Identifying susceptibility factors within distinct phenotypic subgroups is an important advance to understanding the variable susceptibility of COPD in general and sex-specific features specifically. Longitudinal studies following changes in emphysema will be necessary to clarify sex-specific effects of cigarette smoke on emphysema development and progression and potentially to identify additional sex-specific factors contributing to irreversible parenchymal lung destruction.

\section{Conclusions}

Among subgroups of non-Hispanic white and African-American smokers, including those with advanced COPD, early-onset COPD and severe emphysema, males and females demonstrate equivalent emphysema despite significantly fewer pack-years of cigarette smoking among females. COPD is a heterogeneous disease and identifying phenotypically distinct subsets remains an important step in our understanding of the sexually dimorphic features of disease pathogenesis. Continued research on sex differences in COPD is mandatory, with a hope to proceed toward sex-specific primary prevention, diagnostics and therapeutics.

\section{Acknowledgements}

The authors would like to thank participants and field investigators in COPDGene for their willingness to contribute to medical research.

COPDGene Investigators: Core Units. Administrative Core: James Crapo (Principal Investigator), Edwin Silverman (Principal Investigator), Barry Make, Elizabeth Regan. Genetic Analysis Core: Terri Beaty, Nan Laird, Christoph Lange, Michael Cho, Stephanie Santorico, John Hokanson, Dawn DeMeo, Nadia Hansel, Craig Hersh, Peter Castaldi, Merry-Lynn McDonald, Emily Wan, Megan Hardin, Jacqueline Hetmanski, Margaret Parker, Marilyn Foreman, Brian Hobbs, Robert Busch, Adel El-Bouiez, Peter Castaldi, Megan Hardin, Dandi Qiao, Elizabeth Regan, Eitan Halper-Stromberg, Ferdouse Begum, Sungho Won, Brittney Fredericksen, Sharon Lutz. Imaging Core: David A. Lynch, Harvey O. Coxson, MeiLan K. Han, Eric A. Hoffman, Stephen Humphries, Francine L. Jacobson, Philip F. Judy, Ella A. Kazerooni, John D. Newell Jr, Elizabeth Regan, James C. Ross, Raul San Jose Estepar, Berend C. Stoel, Juerg Tschirren, Eva van Rikxoort, Bram van Ginneken, George Washko, Carla G. Wilson, Mustafa Al Qaisi, Teresa Gray, Alex Kluiber, Tanya Mann, Jered Sieren, Douglas Stinson, Joyce Schroeder, Edwin Van Beek. PFT QA Core, Salt Lake City, UT, USA: Robert Jensen. Data Coordinating Center and Biostatistics, National Jewish Health, Denver, CO, USA: Douglas Everett, Anna Faino, Matt Strand, Carla Wilson. Epidemiology Core, University of Colorado Anschutz Medical Campus, Aurora, CO, USA: John E. Hokanson, Jennifer Black-Shinn, Gregory Kinney, Sharon Lutz, Katherine Pratte.

COPDGene Investigators: Clinical Centers. Ann Arbor VA, MI, USA: Jeffrey Curtis, Carlos Martinez, Perry G. Pernicano. Baylor College of Medicine, Houston, TX, USA: Nicola Hanania, Philip Alapat, Venkata Bandi, Mustafa Atik, Aladin Boriek, Kalpatha Guntupalli, Elizabeth Guy, Amit Parulekar, Arun Nachiappan. Brigham and Women's Hospital, Boston, MA, USA: Dawn DeMeo, Craig Hersh, George Washko, Francine Jacobson. Columbia University, New York, NY, USA: R. Graham Barr, Byron Thomashow, John Austin, Belinda D'Souza, Gregory D.N. Pearson, Anna Rozenshtein. Duke University Medical Center, Durham, NC, USA: Neil MacIntyre Jr, Lacey Washington, H. Page McAdams. Health Partners Research Foundation, Minneapolis, MN, USA: Charlene McEvoy, Joseph Tashjian. Johns Hopkins University, Baltimore, MD, USA: Robert Wise, Nadia Hansel, Robert Brown, Karen Horton, Nirupama Putcha. Los Angeles Biomedical Research Institute at Harbor UCLA Medical Center, Los Angeles, CA, USA: Richard Casaburi, Alessandra Adami, Janos Porszasz, Hans Fischer, Matthew Budoff, Dan Cannon, Harry Rossiter. Michael E. DeBakey VAMC, Houston, TX, USA: Amir Sharafkhaneh, Charlie Lan. Minneapolis VA, MN, USA: Christine Wendt, Brian Bell. Morehouse School of Medicine, Atlanta, GA, USA: Marilyn Foreman, Gloria Westney, Eugene Berkowitz. National Jewish Health, Denver, CO, USA: Russell Bowler, David Lynch. Reliant Medical Group, Worcester, MA, USA: Richard Rosiello, David Pace. Temple University, Philadelphia, PA, USA: Gerard Criner, David Ciccolella, Francis Cordova, Chandra Dass, Robert D’Alonzo, Parag Desai, Michael Jacobs, Steven Kelsen, Victor Kim, A. James Mamary, Nathaniel Marchetti, Aditti Satti, Kartik Shenoy, Robert M. Steiner, Alex Swift, Irene Swift, Gloria Vega-Sanchez. University of Alabama, Birmingham, AL, USA: Mark Dransfield, William Bailey, J. Michael Wells, Surya Bhatt, Hrudaya Nath. University of California, San Diego, CA, USA: Joe Ramsdell, Paul Friedman, Xavier Soler, Andrew Yen. University of Iowa, Iowa City, IA, USA: Alejandro Cornellas, John Newell Jr, Brad Thompson. University of Michigan, Ann Arbor, MI, USA: MeiLan Han, Ella Kazerooni, Fernando Martinez. University of Minnesota, Minneapolis, MN, USA: Joanne Billings, Tadashi Allen. University of Pittsburgh, Pittsburgh, PA, USA: Frank Sciurba, Divay Chandra, Joel Weissfeld, Carl Fuhrman, Jessica Bon. University of Texas Health Science Center at San Antonio, San Antonio, TX, USA: Antonio Anzueto, Sandra Adams, Diego Maselli-Caceres, Mario E. Ruiz.

\section{References}

1 Diaz-Guzman E, Mannino DM. Epidemiology and prevalence of chronic obstructive pulmonary disease. Clin Chest Med 2014; 35: 7-16.

2 Mannino DM, Homa DM, Akinbami LJ, et al. Chronic obstructive pulmonary disease surveillance - United States, 1971-2000. MMWR Surveill Summ 2002; 51: 1-16.

3 Thun MJ, Carter BD, Feskanich D, et al. 50-year trends in smoking-related mortality in the United States. $N$ Engl J Med 2013; 368: 351-364.

4 Dransfield MT, Davis JJ, Gerald LB, et al. Racial and gender differences in susceptibility to tobacco smoke among patients with chronic obstructive pulmonary disease. Respir Med 2006; 100: 1110-1116.

5 Sorheim IC, Johannessen A, Gulsvik A, et al. Gender differences in COPD: are women more susceptible to smoking effects than men? Thorax 2010; 65: 480-485. 
Gan WQ, Man SF, Postma DS, et al. Female smokers beyond the perimenopausal period are at increased risk of chronic obstructive pulmonary disease: a systematic review and meta-analysis. Respir Res 2006; 7: 52.

7 Prescott E, Bjerg AM, Andersen PK, et al. Gender difference in smoking effects on lung function and risk of hospitalization for COPD: results from a Danish longitudinal population study. Eur Respir J 1997; 10: 822-827.

8 Han MK, Postma D, Mannino DM, et al. Gender and chronic obstructive pulmonary disease: why it matters. Am J Respir Crit Care Med 2007; 176: 1179-1184.

9 Nakano Y, Muro S, Sakai H, et al. Computed tomographic measurements of airway dimensions and emphysema in smokers. Correlation with lung function. Am J Respir Crit Care Med 2000; 162: 1102-1108.

10 Timmins SC, Diba C, Farrow CE, et al. The relationship between airflow obstruction, emphysema extent, and small airways function in COPD. Chest 2012; 142: 312-319.

11 Diaz AA, Morales A, Diaz JC, et al. CT and physiologic determinants of dyspnea and exercise capacity during the six-minute walk test in mild COPD. Respir Med 2013; 107: 570-579.

12 Baldi S, Miniati M, Bellina CR, et al. Relationship between extent of pulmonary emphysema by high-resolution computed tomography and lung elastic recoil in patients with chronic obstructive pulmonary disease. Am J Respir Crit Care Med 2001; 164: 585-589.

13 Diaz AA, Bartholmai B, San Jose Estepar R, et al. Relationship of emphysema and airway disease assessed by CT to exercise capacity in COPD. Respir Med 2010; 104: 1145-1151.

14 Spruit MA, Watkins ML, Edwards LD, et al. Determinants of poor 6-min walking distance in patients with COPD: the ECLIPSE cohort. Respir Med 2010; 104: 849-857.

15 Han MK, Kazerooni EA, Lynch DA, et al. Chronic obstructive pulmonary disease exacerbations in the COPDGene study: associated radiologic phenotypes. Radiology 2011; 261: 274-282.

16 Johannessen A, Skorge TD, Bottai M, et al. Mortality by level of emphysema and airway wall thickness. Am J Respir Crit Care Med 2013; 187: 602-608.

17 Haruna A, Muro S, Nakano Y, et al. CT scan findings of emphysema predict mortality in COPD. Chest 2010; 138: 635-640.

18 Dawkins PA, Dowson LJ, Guest PJ, et al. Predictors of mortality in alpha1-antitrypsin deficiency. Thorax 2003; 58: 1020-1026.

19 Camp PG, Coxson HO, Levy RD, et al. Sex differences in emphysema and airway disease in smokers. Chest 2009; 136: $1480-1488$

20 Dransfield MT, Bailey WC. COPD: racial disparities in susceptibility, treatment, and outcomes. Clin Chest Med 2006; 27: 463-471.

21 Martinez FJ, Curtis JL, Sciurba F, et al. Sex differences in severe pulmonary emphysema. Am J Respir Crit Care Med 2007; 176: 243-252.

22 Tatsumi K, Kasahara Y, Kurosu K, et al. Clinical phenotypes of COPD: results of a Japanese epidemiological survey. Respirology 2004; 9: 331-336.

23 Hansel NN, Washko GR, Foreman MG, et al. Racial differences in CT phenotypes in COPD. COPD 2013; 10: 20-27.

24 Clayton JA, Collins FS. Policy: NIH to balance sex in cell and animal studies. Nature 2014; 509: $282-283$.

25 Regan EA, Hokanson JE, Murphy JR, et al. Genetic epidemiology of COPD (COPDGene) study design. COPD 2010; 7: 32-43

26 Foreman MG, Zhang L, Murphy J, et al. Early-onset chronic obstructive pulmonary disease is associated with female sex, maternal factors, and African American race in the COPDGene Study. Am J Respir Crit Care Med 2011; 184: 414-420

27 Diaz AA, Come CE, Ross JC, et al. Association between airway caliber changes with lung inflation and emphysema assessed by volumetric CT scan in subjects with COPD. Chest 2012; 141: 736-744.

28 Gevenois PA, de Maertelaer V, De Vuyst P, et al. Comparison of computed density and macroscopic morphometry in pulmonary emphysema. Am J Respir Crit Care Med 1995; 152: 653-657.

29 Cho MH, Castaldi PJ, Hersh CP, et al. A genome-wide association study of emphysema and airway quantitative imaging phenotypes. Am J Respir Crit Care Med 2015; 192: 559-569.

30 Yuan R, Mayo JR, Hogg JC, et al. The effects of radiation dose and CT manufacturer on measurements of lung densitometry. Chest 2007; 132: 617-623.

31 Dransfield MT, Washko GR, Foreman MG, et al. Gender differences in the severity of CT emphysema in COPD. Chest 2007; 132: 464-470.

32 Silverman EK, Speizer FE, Weiss ST, et al. Familial aggregation of severe, early-onset COPD: candidate gene approaches. Chest 2000; 117: 5 Suppl. 1, 273S-274S

33 Silverman EK, Mosley JD, Palmer LJ, et al. Genome-wide linkage analysis of severe, early-onset chronic obstructive pulmonary disease: airflow obstruction and chronic bronchitis phenotypes. Hum Mol Genet 2002; 11: 623-632.

34 Silverman EK, Palmer LJ, Mosley JD, et al. Genomewide linkage analysis of quantitative spirometric phenotypes in severe early-onset chronic obstructive pulmonary disease. Am J Hum Genet 2002; 70: 1229-1239.

35 DeMeo DL, Carey VJ, Chapman HA, et al. Familial aggregation of FEF25-75 and FEF25-75/FVC in families with severe, early onset COPD. Thorax 2004; 59: 396-400.

36 Silverman EK, Weiss ST, Drazen JM, et al. Gender-related differences in severe, early-onset chronic obstructive pulmonary disease. Am J Respir Crit Care Med 2000; 162: 2152-2158.

37 Wang Y, Beydoun MA. The obesity epidemic in the United States - gender, age, socioeconomic, racial/ethnic, and geographic characteristics: a systematic review and meta-regression analysis. Epidemiol Rev 2007; 29 : 6-28.

38 Ogawa E, Nakano Y, Ohara T, et al. Body mass index in male patients with COPD: correlation with low attenuation areas on CT. Thorax 2009; 64: 20-25.

39 Castaldi PJ, San Jose Estepar R, et al. Distinct quantitative computed tomography emphysema patterns are associated with physiology and function in smokers. Am J Respir Crit Care Med 2013; 188: 1083-1090.

40 Galban CJ, Han MK, Boes JL, et al. Computed tomography-based biomarker provides unique signature for diagnosis of COPD phenotypes and disease progression. Nat Med 2012; 18: 1711-1715.

41 Camp PG, Ramirez-Venegas A, Sansores RH, et al. COPD phenotypes in biomass smoke- versus tobacco smoke-exposed Mexican women. Eur Respir J 2014; 43: 725-734. 